\title{
Ist Kreativität ein subjektives oder ein kollektives Phänomen? Über eine ungeklärte Frage in der Non-representational Theory und eine denkbare Antwort der Phillosophie der symbolischen Formen
}

\author{
P. Dirksmeier \\ Humboldt-Universität zu Berlin, Geographisches Institut, Unter den Linden 6, 10099 Berlin, Germany \\ Correspondence to: P. Dirksmeier (peter.dirksmeier@geo.hu-berlin.de)
}

Received: 24 July 2012 - Revised: 21 January 2014 - Accepted: 28 January 2014 - Published: 8 October 2014

Kurzfassung. The micro-macro-problem of the social sciences is also present in human geographies' discussions of creativity. Creativity could be conceptualized either as a capability of subjects or as emerging from interaction processes. A direct consequence of this theoretical indecision is an inconsistent notion of creativity in Nigel Thrifts' nonrepresentational theory (NRT) that is originally developed to valuate creative praxis. The paper advances a proposal for conceptualizing creativity in NRT by using the philosophy of symbolic forms established by Ernst Cassirer. First, the paper develops a notion of individual creativity that is implied in Cassirers' work on symbolic pregnance and symbolic forms and via Bourdieus' interpretation of Cassirer also in the French sociologists' theory of incorporation. Second, the paper shows two possibilities to include the outlined notion of individual creativity in NRT. Creativity could be linked with NRT by modifying Bourdieus' concept of incorporation as used to establish a notion of nonrepresentational praxis. The second possibility of including creativity is a connection of affect and experience, which leads directly to the emergence of novelty.

\section{Fragestellung}

Ungelöste theoretische Probleme der Nachbarwissenschaften stellen die Humangeographie häufig selbst vor konzeptionelle Herausforderungen. Eine solche offene Frage in der Sozialwissenschaft, die in besonderer Weise die Humangeographie betrifft, ist die Verbindung individuellen Handelns mit dem kollektivistischen Phänomen der Gesellschaft, auch Mikro-Makro-Problem oder Mikro-Makro-Verhältnis genannt (Heintz, 2004). Ein prominenter Diskurs, der unmittelbar durch diese Spannung von Individualisierung auf der einen, Vergesellschaftung und Globalisierung auf der anderen Seite tangiert wird, ist die geographieinterne Diskussion um Kreativität. Die Kreativitätssemantik spaltet sich gleichsam in eine individuelle und eine kollektive Annäherung an das Phänomen der Entstehung von Neuheit im Allgemeinen, und dies ist einer der Gründe für die Unklarheit bezüglich ihres Gegenstandes.
In der Humangeographie findet sich überwiegend eine Auffassung von Kreativität, die vor allem Interaktionen in Milieus, Netzwerken oder Projekten als wesentlich für die Entstehung von neuen Ideen oder Produkten ansieht (z.B. Grabher, 2001; Törnqvist, 2004). Zwar erkennt Meusburger allein ,more than one hundred different definitions of creativity" (Meusburger, 2009:99), die jedoch überwiegend dieselben Attribute anführen und nicht primär Individuen adressieren. Kreativität ist vornehmlich mit Neuheit, Überraschung und Pragmatik assoziiert (Meusburger, 2009:99), die wiederum als Folge von Interaktion entstehen. Gleichzeitig bleibt die Rolle der individuellen Kreativität in diesen Interaktionssystemen ungeklärt. Diese Unklarheit provoziert jedoch in der gegenwärtigen Theoriebildung in der Humangeographie wiederum Probleme, wie sich am Beispiel der vieldiskutierten Non-representational theory (NRT) und ihres Kreativitätsverständnisses aufzeigen lässt.

In der klassischen Kreativitätsdiskussion werden dagegen früh auf individualtheoretischen Grundlagen beruhende 
kognitive Ansätze verwendet, um Kreativität näher zu bestimmen. Wallas Vier-Stufenmodell der individuellen Kreativität, das aus der zeitlichen Abfolge von Präparationsphase als Vorbereitung des kreativen Aktes, Inkubationsphase als Entwicklung einer Idee, Illuminationsphase als Bewusstwerdung dieser neuen Idee und deren Begleiterscheinungen und der Verifikationsphase als Überprüfung des Resultats besteht (Wallas, 1926:10), ist eine frühe Beschreibung der individuellen Kreativität. Whiteheads 1929 erstmals publizierter prozesshafter Ansatz, der Kreativität als eine plötzliche oder sukzessive Einführung von Veränderung in einen Kontext von gleichen Entitäten begreift, entstand zeitgleich mit Wallas Modell (Whitehead, 1979:21). Das Problem der Operationalisierung von individueller Kreativität diskutiert Guilford. Dieser erkennt ,,rhythms of creativity“ (Guilford, 1950:445), die dafür Sorge tragen, dass die Zuschreibung kreativer Leistungen auf ein Subjekt nicht jederzeit möglich ist. Zudem verwischen Konformitätsnormen, die vor allem in der Sozialisationsphase greifen, Zuschreibungen individueller Kreativität (Guilford, 1967:334). Es besteht demnach eine Spannung zwischen sozial erwartetem und abweichendem Denken.

Einen deutlichen Schritt in Richtung des Einbezugs der sozialen Bedingungen der Umwelt eines Subjekts in die Erfassung von individueller Kreativität leistet Rhodes mit seinem 4P-Modell. Individuelle Kreativität speist sich demnach aus den vier Faktoren der Person in ihren Eigenschaften als menschliches Wesen (person), mentaler Prozesse, die operativ neue Ideen hervorbringen (process), des ökologischen Drucks aus der Umwelt auf die Person, u.a. aufgrund der Existenz der anderen Subjekte (press), sowie sprachlicher und werklicher Ideen, die die Produkte der Kreativität repräsentieren (products) (Rhodes, 1961). Csikszentmihalyi betont dagegen sowohl die Bedeutung der intrinsischen Motivation für individuelle Kreativität (Csikszentmihalyi, 1997:107) als auch der Aufmerksamkeit einer relevanten sozialen Gruppe für das Ergebnis des kreativen Akts (Csikszentmihalyi, 1988:168). Diese bei Rhodes und Csikszentmihalyi anklingende Bedeutung des sozialen Kontexts für individuelle Kreativität führt Amabile in ihrem Entwurf einer Sozialpsychologie der Kreativität weiter aus (Amabile, 1996). Kaufman und Beghetto entwickeln schließlich mit dem 4C-Modell eine Spezifizierung individueller Kreativität in die hierarchischen Stufen „mini-c“ als individuelle Interpretationen von Erfahrungen und Handlungen, „little-c" als Alltagskreativität, „Pro-C“ als Zwischenschritt von Alltagskreativität zum Expertentum und „Big-C“ als professionalisierte und anerkannte Kreativität (Kaufman und Beghetto, 2009).

In der Kreativitätsdiskussion spiegelt sich letztlich das Mikro-Makro-Problem bzw. -Verhältnis der Sozialwissenschaft wider. Ein bedeutendes Forschungsdesiderat liegt hier in der Beziehung von individueller Kreativität zu ihrer kollektiven Bedingung (Taggar, 2002), oder anders formuliert, wie Individuen in Kollektiven und Interaktionssy- stemen, z.B. Organisationen oder Projekten, kreativ sind, welche Ideen und Produkte individuell und welche kontextuell hervorgebracht werden (Chen, 2012). Diese MikroMakro-Unterscheidung tangiert ebenfalls mit der NRT eine Theorie in der Humangeographie, die, obwohl sie das Jetzt, das nicht-repräsentierbare kontingente Gegenwärtige thematisiert, nicht überzeugend darlegen kann, wie Neues entsteht. Zum einen resultiert dieses Theorieproblem aus der konzeptionellen Substituierung des Subjekts durch ,individuating affective states of an anonymous force" (Spinoza zit. in Thrift, 2008:13) in der NRT, die sich folglich nicht von Arbeiten zum kreativen Subjekt inspirieren lässt. Zum anderen gelingt diese Ersetzung des Subjektes in der NRT nicht konsequent, und in der Folge schwingt eine schwammige subjektive Rest-Kreativität mit, die radikal kollektiviert und in einem poststrukturalistischen Zwischen-Raum von Affekten, Netzwerken, Flüssen und Akteurs-ObjektKonstellationen ausgelagert wird (Wylie, 2010:101).

Vor diesem Hintergrund ist das Ziel des Beitrages die Verdeutlichung dieses weitgehend unbeachteten Theorieproblems der NRT (Wylie, 2010), die nicht plausibilisieren kann, ob Kreativität ein individuell gebundenes Phänomen ist oder einen Emergenzeffekt darstellt. Der Aufsatz erarbeitet darüber hinaus einen Vorschlag, wie ein konzeptioneller Weg, der aus diesem Problem weist, aussehen könnte. Einen solchen möglichen Ausweg erkennt der Beitrag in der Philosophie der symbolischen Formen (PSF) Ernst Cassirers, der sich einer tiefgreifenden Analyse des Verhältnisses von Einheit und Vielheit, Individuum und symbolischen Formen oder modern-sozialtheoretisch gewendet, des MikroMakro-Problems widmet. Cassirer bietet für eine Verbindung mit dem Subjekt/Kreativitätsproblem der NRT zwei Vorteile. Cassirers Konzeption des menschlichen Geistes impliziert bereits Kreativität (Verene, 1978:17). Mit seiner Idee der Verwirklichung der menschlichen geistigen Identität im „wirkenden Handeln[...] als eines schöpferischen Gestaltens" (Schwemmer, 1997:28), die Cassirer etwa in seinen drei Aufsätzen über Goethe darlegt (Cassirer, 1932), liefert er darüber hinaus eine denkbare Konzeption von individueller Kreativität im Kontext seiner Arbeiten zu den symbolischen Formen als „Grundformen des ,Verstehens' der Welt“ (zit. nach Krois, 1988:19). Darüber hinaus fokussiert Cassirers Arbeit über die symbolischen Formen das Verhältnis von Individuum zu kollektiven Bedingungen. Diese Analyse wird von Bourdieu sozialtheoretisch weitergeführt und auf das Verhältnis von individuellen Praktiken, Geschmack und gesellschaftlichen Positionierungen angewendet (Bourdieu, 1987a; 1991:629) und findet wiederum über die Figur der Inkorporierung Eingang in die NRT (Thrift, 1996:2).

Aufbauend auf einer Darlegung der relevanten Inhalte der Philosophie der symbolischen Formen im folgenden Kapitel (Kapitel 2) lotet der vorliegende Beitrag die Möglichkeiten dieser Philosophietradition für die Erarbeitung einer Lösung des Kreativitätsproblems der NRT aus (Kapitel 3). Die in der NRT gewählte Strategie der Konzipierung von 
Kreativität als ein Kollektivbegriff von Affekten, Objekten, Netzwerken und Performanzen sowie die mit dieser Auffassung verbundene theoretische Problematik wird in Kapitel 4 dargelegt. Der Beitrag schließt mit einer Synthese von individueller Kreativität im Sinne Cassirers und der NRT und damit mit einer Spezifizierung eines besonderen Mikro-MakroVerhältnisses für die humangeographische Theoriebildung (Kapitel 5).

\section{Symbolische Form und symbolische Prägnanz - zwei Schlüsselbegriffe im Werk Cassirers}

Die PSF entspinnt sich um das Verhältnis der Vielfalt objektiver symbolischer Ausprägungen auf der einen und der Einheit des subjektiven menschlichen Geistes auf der anderen Seite (Schwemmer, 1997:24). Für Cassirer besteht eine enge Verbindung von objektiver Erkenntnis und intersubjektiver und subjektiver Deutung der Welt (Paetzold, 2008:8). Die PSF nimmt damit die skizzierte theoretische Problematik der Kreativitätsdiskussion in gewisser Weise vorweg und bietet sich als Theorie an, dieses Problem vertieft zu denken. Cassirers Theorieverständnis geht dabei weit über die Sphäre wissenschaftlicher Erkenntnis hinaus. Theorie findet sich überall dort, ,wo überhaupt eine spezifische Weise der Gestaltung, der Erhebung zu einer bestimmten Einheit des ,Sinnes“ am Werke ist" (Cassirer, 2010:18). Nach Schwemmer (1997:23-24) sind für die Theorie der PSF drei Aspekte wesentlich. Zunächst ist die wissenschaftliche Erkenntnis in der PSF gegenüber der subjektiven Welterfassung weder privilegiert noch ein Primus inter Pares, sondern eine von vielen verschiedenen möglichen Auffassungsweisen. Diese wiederum verknüpfen sich zweitens mit den symbolischen Formen als unterscheidbare, irreduzible und ,bestimmte geistige Auffassungsweise[n]“ (Cassirer, 1994a:9), die „zugleich eine eigene Seite des ,Wirklichen““ (Cassirer, 1994a:9) gründen. Dieses sinnliche Wahrnehmen beruht drittens auf der ,emotionale[n] Dimension der menschlichen Existenz" (Schwemmer, 1997:24), die Cassirer mit „Gefühlsgrund“ (Schwemmer, 1997:24) bezeichnet. Die PSF ist letztlich Cassirers Versuch, die Pluralität menschlicher Auffassungen in ein bestimmtes Modell der Bedeutungskonstitution zu bündeln, auf das sich alle Formen der sinnlichen Wahrnehmung reduzieren lassen (Neher, 2005). Cassirer selbst sieht die Zahl der symbolischen Formen als endlich an und hat Mythos, Sprache, Technik, Wissenschaft, Kunst, Geschichte, Religion, Sitte, Recht und Wirtschaft als symbolische Formen ausgearbeitet oder erwähnt (Krois, 1984:440; Paetzold, 2008:43). Dagegen betont Verene (1969:45), dass die Zahl möglicher symbolischer Formen potenziell nicht limitiert sei.

In Cassirers Symbolauffassung kann ein Symbol eine Handlung, ein Klang oder ein Objekt sein, in dem jeweils eine Bedeutung involviert ist (Verene, 1978:17). Symbole sind demnach reine Verbindungen zwischen dem Materiellen und dem Ideellen und substituieren nicht das Ideelle durch das
Materielle. Cassirer sieht im Symbolischen den ideellen oder logischen Aspekt immer durch etwas Sinnliches ausgedrückt (Hoel, 2011:81). Cassirer definiert selbst seine Symbolauffassung wie folgt. „Wir versuchten mit ihm [dem Symbolbegriff] das Ganze jener Phänomene zu umfassen, in denen überhaupt eine wie immer geartete ,Sinnerfüllung' des Sinnlichen sich darstellt - in denen ein Sinnliches, in der Art seines Daseins und Soseins, sich zugleich als Besonderung und Verkörperung, als Manifestation und Inkarnation eines Sinnes darstellt" (Cassirer, 2010:105). Das Symbol kennzeichnet nach dieser Auffassung eine besondere Beziehung von Sinn und Sinnlichkeit. Sämtlicher Sinn findet sich im Medium des Sinnlichen wieder (Paetzold, 2008:39). Cassirer betont die Ambivalenz des Symbols, das zum einen ein „Haften am Sinnlichen" (Cassirer, 1995:2) genauso kennzeichne wie ein „Hinausgehen über das Sinnliche“ (Cassirer, 1995:2). Die sich im Symbol ausdrückende Beziehung von Sinn und Sinnlichkeit, dem Ideellen und dem Materiellen, folgt dabei einem grundlegenden Prinzip, dessen Aufdeckung als ,ein in sich geschlossenes und einheitliches Grundverfahren" (Cassirer, 2009:66) der Symbolisierung ein Ziel der PSF darstellt.

Symbolische Form bedeutet nun eine bestimmte Form der Weltdeutung, die an der Grenze von Sinn und Sinnlichem entsteht. Die verschiedenen symbolischen Formen sind untereinander gleichrangig konzipiert und lassen sich nicht aufeinander reduzieren. Jede symbolische Form bezeichnet eine unabhängige Entität (Verene, 1969:45). Die häufig zitierte (etwa bei Krois, 1984:440; Krois, 1988:19; Paetzold, 2008:41) Definition von symbolischer Form bei Cassirer lautet: „Unter einer ,symbolischen Form“ soll jede Energie des Geistes verstanden werden, durch welche ein geistiger Bedeutungsgehalt an ein konkretes sinnliches Zeichen geknüpft und diesem Zeichen innerlich zugeeignet wird“" (Cassirer, 2009:67). Die symbolische Form besteht demnach aus dem sinnlichen Zeichen, dem Sinn und einer „Energie des Geistes“ als Rückgriff auf Wilhelm von Humboldts Unterscheidung von „Ergon“ und „Energeia“ in der Sprache (Krois, 1984:440; Paetzold, 2008:41). Bei de Saussure entspricht dies der Unterscheidung von „langue“ und „parole“, bei Chomsky von „,competence“ und „performance“. Die Unterscheidung bezieht sich auf zwei Formen der Sprache als abgeschlossenes System auf der einen und modifizierendes Sprechen und Schreiben auf der anderen Seite (Krois, 1984:440; Paetzold, 2008:41). Die symbolische Form vollbringt damit eine Übersetzungsleistung in der Art, dass reine Eindrücke und Wahrnehmungen des Bewusstseins mit dem symbolisch-sinnhaften Inhalt verbunden werden (Cassirer, 2009:69). Damit wird der Begriff in der PSF zweifach verwendet. Er bezeichnet sowohl alles, was „eine symbolische Form erfüllt“ (Krois, 1984:440), als auch einen „Interpretationszusammenhang“ (Krois, 1984:440), der auf sämtliche denkbaren Objekte angewendet werden kann. Diese Breite des Begriffs führt dazu, dass symbolische Form bereits mit Kultur gleichgesetzt wurde (Heusden, 2003:124). 
Cassirer spricht von der symbolischen Prägnanz, die den Dingen innewohnt und dafür Sorge trägt, dass sich die Verschiedenheit der Sinneseindrücke der menschlichen Wahrnehmung in erkennbare und begreifbare Formen bündelt (Mersch, 2006:351). Nach Krois ist symbolische Prägnanz der grundlegendste Begriff in der PSF, da er sich nicht auf Zeichen stützt, die wiederum ,eine fundamentalere Art der Bedeutung“ (Krois, 1988:22) voraussetzen. Vielmehr bezeichnet symbolische Prägnanz die Grundlage aller menschlichen Erfahrung. Symbolische Prägnanz ist bei Cassirer definiert als ,die Art [...], in der ein Wahrnehmungserlebnis als , sinnliches' Erlebnis, zugleich einen bestimmten nichtanschaulichen ,Sinn“ in sich faßt und ihn zur unmittelbaren konkreten Darstellung bringt" (Cassirer, 2010:231). Symbolische Prägnanz ist eine anthropologische Konstante, die die Wahrnehmung von Sinn ,als Bedingung der Möglichkeit von Erfahrung“ (Krois, 1984:441) bezeichnet. Der Begriff ist irreduzibel und zirkulär. Kein Beweis kann die symbolische Prägnanz begründen, da ein solcher wiederum über symbolische Prägnanz gewonnene Erfahrung zu ihrer Erklärung voraussetzt (Krois, 1984:441).

\section{Kreativität im Kontext symbolischer Formen}

Wie lässt sich nun individuelle Kreativität in der PSF verorten? In den Arbeiten Cassirers zum Erkenntnisproblem findet sich eine Auffassung von Wahrnehmung, die diese als Bestand und Veränderung von Sinneseindrücken begreift (Cassirer, 1944). Die Wahrnehmung vollzieht sich in einem Spannungsfeld von Bestand und Veränderung. Symbolische Prägnanz und symbolische Form bieten hierfür eine Ausarbeitung. Die symbolische Prägnanz als Grundlage sinnhafter menschlicher Erfahrung und damit auch der Kommunikation von Bedeutungen transformiert das Wahrgenommene im subjektiven Akt der Wahrnehmung als Interpretation von Zeichen und Bezeichnetem im Kontext symbolischer Formen. Die subjektive Wahrnehmung ist damit keine reine Reproduktion eines Draussen, sondern ein Prozess der Objektivierung (Cassirer, 1944:19-20) subjektiver sinnhafter Erfahrung und weist somit ein Moment der Kontingenz auf.

Cassirer folgt in der PSF einer Idee des Subjekts, die er als „Ich“ tituliert und das über symbolische Prägnanz sinnlich erfährt. Das Ich ist das letztmögliche, reine Subjektive in der Philosophie Cassirers. Es steht im Gegensatz zum Objektiven und Dinghaften, symbolisch dem wahrnehmenden Subjekt Vermittelten. „Der Grundcharakter des reinen Ich besteht darin, daß es, im Gegensatz zu allem Objektiven und Dinghaften, absolute Einheit ist. Das Ich, als reine Form des Bewußtseins gefaßt, enthält keinerlei Möglichkeit innerer Unterschiede mehr" (Cassirer, 1994a:229). Dieses Ich als ein kohärentes Subjekt resultiert aus seinem Tun heraus (Schwemmer, 1997:212). Es ist das Einzelne, Ununterscheidbare und Unteilbare schlechthin, da Unterschiede und Teile in Cassirers Philosophie wiederum der Objektwelt zugehören. Das Ich als Subjekt ist dagegen ,'reine Identität mit sich selbst"“ (Cassierer, 1994a:229).

Cassirers Lösung der Frage der Beziehung des Universellen zum Partikularen, des Objektiven zum Subjektiven besteht nun darin, dass er diese Beziehung selbst als ein fundamentales Prinzip der Kognition ansieht. Das Universelle kann demnach nur im Partikularen wahrgenommen werden. Auf der anderen Seite ist das Partikulare nur in seiner Relation zum Universellen denkbar (Hoel, 2011). Diesen Übergang bezeichnet Cassirer als ,Transformation“ (Cassirer, 1944:13). Eine symbolische Form ist damit eine ganz bestimmte Zeichnung von Welt, die sich über die Objektivierung und mithin Universalisierung von individuellpartikularen Eindrücken und Vorstellungen des Bewusstseins, d.h. des Ichs bzw. Subjekts, als symbolische Prägnanz vollzieht (Magerski, 2005).

In der modernen Sozialwissenschaft greift vor allem Bourdieu auf Cassirers Arbeiten zurück, um seine Lesart des Mikro-Makro-Problems als vom, ,klassischen Rationalismus aufgestellten Unterscheidung zwischen Verstandeswahrheiten und Tatsachenwahrheiten“ (Bourdieu, 1987b:12) zu begründen. Er fügt Cassirers Arbeiten zu symbolischer Prägnanz und symbolischer Form den Aspekt gesellschaftlicher Macht hinzu, um die ,symbolische Ordnung“ (Bourdieu, 2001:216) der Gesellschaft zu erklären. Bourdieus Grundbegriffe des Habitus und Feldes sind als soziologische Weiterentwicklungen der grundlegenden Ideen der symbolischen Prägnanz und symbolischen Form interpretierbar (Bickel, 2003:113). Cassirers Philosophie ist damit eine implizite theoretische Grundlage der modernen Sozialwissenschaft.

Symbolische Formen ruhen auf der Fähigkeit des menschlichen Geistes, empirische Entitäten als seriell geordnet zu begreifen, wobei diese Serien wiederum bestimmten nachvollziehbaren Regeln unterliegen (Verene, 1978:15). Der Mensch gestaltet in diesem Sinne die Realität und in diesem Tun formt sich das Subjekt aus. „Und nur in diesem Bilde der gestalteten Erlebniswirklichkeit findet er [der Mensch] sodann sich selbst als ,Subjekt', als monadischen Mittelpunkt des vielgestaltigen Daseins wieder" (Cassirer, 2010:101). Das Subjekt als ein gestaltendes Ich greift auf die symbolischen Formen zu, die als Modi der Strukturierung von Erfahrung Kategorien des Denkens wie Grund, Objekt, Ding, Eigenschaft, Raum, Zeit, Zahlen und Selbst ordnen (Verene, 1978:18).

Die Signifikanz der individuellen Kreativität eines Subjekts als gestaltendes Ich in der cassirerschen Philosophie findet sich in der Tatsache, dass das menschliche Denken von einer Theorie der Kognition über die symbolischen Formen zu einer Theorie der Kultur ausgedehnt wird (Verene, 1978:19). Cassirer verdeutlicht dies in seinem Aufsatz zur „Tragödie der Kultur“, in dem ,der schöpferische Prozess““ (Cassirer, 1994b:110) als kreativer Prozess des subjektiven Tuns zu einer Grundlage der Kultur erhoben wird. Die individuelle Kreativität ist in Cassirers Philosophie die Basis der Kultur, die mithilfe der symbolischen Formen vermittelt 
wird. „Der Mensch hat in den ,symbolischen Formen“, die das Eigentümliche seines Wesens und seines Könnens sind, gewissermaßen die Lösung einer Aufgabe vollzogen, die die organische Natur als solche nicht zu lösen vermochte. [...] Hier ist das Werden und Wirken des einzelnen in ganz anderer, tief eingreifender Weise mit dem des Ganzen verknüpft. Was die Individuen fühlen, wollen, denken, bleibt nicht in ihnen selbst verschlossen; es objektiviert sich im Werk" (Cassirer, 1994b:126). Die basale Funktion der individuellen Kreativität, d.h. die synthetische kognitive Aktivität eines Subjekts, findet sich im Akt der Symbolisierung, der das subjektive „Tun“ in einen kulturellen Kontext überführt. Verene sieht daher in seiner Lesart einer cassirerschen „theory of creativity" (1978:19) jeden subjektiven kreativen Akt in einer Relation zu den symbolischen Formen einer Kultur.

Die individuelle Kreativität als ein Konzept in der Lesart der PSF erhebt damit die symbolische Prägnanz, d.h. den Zusammenhang von Sinnlichem und Sinn, zu einem Element der Differenzierung zwischen dem Individuellen und dem Allgemeinen. Jedes Ich, dass im schöpferischen Gestalten etwas Neues in die Welt bringt, und dies als kreatives Subjekt mit bestimmten Wahrnehmungen von Sinnlichem und sinnhaften Interpretationen verbindet, tut dies in Relation zu einer Kultur, die als Kontext erst den schöpferischen Akt oder das schöpferische Gestalten als kreativ und das Ergebnis als neu erscheinen lässt (Verene, 1978:24). Die kreativen Subjekte in ihrem schöpferischen Gestalten werden demnach erst vor einem allgemeinen kulturellen Hintergrund unterscheidbar, wie Verene betont. Die kreativen Subjekte ,are distinguishable from each other by the fact that they follow the directions of the symbolic process in human culture seen as a structure of symbolic forms" (Verene, 1978:28). Individuelle Kreativität in der PSF ist damit definierbar als jede Form von kognitiver Aktivität von Subjekten als Verknüpfungen von Sinnlichem und Sinn, die über die symbolischen Formen in Kultur transformiert wird.

\section{Die Kreativität der NRT}

Ein bedeutendes Ziel des nonrepresentationalen Ansatzes von Thrift ist es, die kreative Praxis wertzuschätzen, wie er selbst in den ersten zwei Sätzen der Zusammenfassung seines paradigmatischen Aufsatzes „Afterwords“ betont. „Gradually, then, it has become clear to me what I am trying to do. I want to provide a body of work which values creative praxis“" (Thrift, 2000a:213). Die im Rahmen der NRT entwickelte Ontologie zielt auf die Praxis im Jetzt sowie auf die Kreativität dieser expressiven Praktiken und nicht auf deren Repräsentation, die nach Thrift (1999) die Grand Theorys bis dato dominiere. Die NRT fragt vielmehr allgemein danach, wie das Mögliche mit dem Möglichen zurechtkomme (Thrift, 2008).

Vor diesem Hintergrund der Bedeutung von kreativer Praxis für die Theorie überrascht der geringe Beitrag der NRT zur Kreativitätsdiskussion. „,[N]on-representational theories have actually had relatively little to say about creativity per $s e$ " (Wylie, 2010:100-101). Das große Potenzial der NRT, bestimmte Formen von Kreativität in Praktiken zu denken und diese Praxisdiskussion wiederum mit einem tragfähigen Begriff der Kreativität zu versorgen, liegt folglich brach. Einen möglichen Grund dieser Situation sieht Wylie in der Tatsache, dass die NRT nicht über das kreative Subjekt selbst nachdenkt und sich folglich nicht von Arbeiten zum Kreativsubjekt inspirieren lässt (Wylie, 2010:101). Kreativität würde vielmehr über Arbeiten in die NRT gebracht, die vor allem die Verwertungsfokussiertheit und Kommodifizierung des Konzeptes in den Vordergrund stellen und kritisieren (z.B. Osborne, 2003). Dennoch ist das (kreative) Subjekt nicht vollständig aus der NRT eliminiert (Wylie, 2010:101), sondern es emergiert über Affekte, Wahrnehmungen (Dewsbury et al., 2002:439) und Performanzen. Das Durchhalten einer dezentralen Perspektive auf das Subjekt als Träger_in der Praxis (Thrift, 1997:127-129) gelingt nicht konsequent. Thrift betont zwar die Bedeutung von Praktiken als Surrogat des Subjekts, ,non-representational theory concentrates [...] on practices, understood as material bodies of work or styles that have gained enough stability over time, $[\ldots]$ to reproduce themselves" (Thrift, 2008:8), dennoch bleibt über die Konzepte der Intentionalität und des impliziten Wissens eine Subjektkonzeption in der Schwebe. Die NRT weist eine versteckte Agenda der Wertschätzung von kognitiven Dimensionen der Intentionalität auf, die in der Tradition der Phänomenologie wurzelt und damit das Subjekt implizit mitführt (Barnett, 2012:382).

Der Begriff des Kreativen bleibt in der NRT unterbestimmt. An einer Stelle betont Thrift, dass Kreativität in ökonomischen Zusammenhängen als ein Wert an sich betrachtet würde. Im Zusammenhang mit der Förderung von Innovationen erfahren diejenigen Prozesse die größte Aufmerksamkeit, die Kreativität in Innovationen umsetzen, wobei Thrift Kreativität mit einer ,,creative“ knowledge“ (Thrift, 2005:133) verbindet ohne näher darauf einzugehen, wo dieses Wissen verortet ist (Thrift, 2005:133). Es könnten Subjekte genauso Träger_innen von Kreativität sein, wie Netzwerke oder Prozesse. An anderer Stelle sieht er ,innovation and creativity as moments of ,intuition “" (Thrift, 2006:192) an und betont deren Kontingenz. Mit dieser allgemeinen Aussage bleibt die Adresse der Kreativität ebenfalls unklar. Als ein Aspekt der Kreativitätsauffassung, die der NRT zugrunde liegt, lässt sich hingegen eine unspezifische Interdependenz von Wissen, implizitem Wissen, Intuition, Spiel und Performanz bzw. expressiven Handeln festhalten (Thrift, 2008:32;119).

Wie konzipiert die NRT nun Kreativität im Spannungsfeld von individuellen und kollektiven Ansätzen? Wylie skizziert drei verschiedene theoretische Spielarten, in denen Kreativität in der NRT vorkommt, die sämtlich Kollektivkonzepte abbilden (Wylie, 2010:104). Diese drei theoretischen Orte halten bei einer genaueren Prüfung ihre subjektlose Position 
allerdings nicht durch. Zwei dieser drei Ansätze bleiben letztlich unentschieden bei der Frage, ob Kreativität ein subjektiv gebundenes oder ein Emergenzphänomen darstellt.

Der erste theoretische Ansatz der Kreativität in der NRT ist für Wylie die Philosophie Deleuzes, der das Kreative als bedeutsam einstufe, allerdings nicht in einem zentralen Punkt des Subjektes kulminieren ließe. Kreativität komme bei Deleuze ohne Subjektivität aus. „Deleuze of course emphasises creativity, and cognate notions of invention, experimentation, connectivity and vitality; these are some of the key motifs of a philosophy of becoming and transformation [...] this is creativity without subjectivity - creativity without either a subject-who-creates, or a subject-created as an end-point or culminating moment" (Wylie, 2010:104). Thrift selbst distanziert sich allerdings von dieser deleuzeischen Kreativitätsvorstellung. Zwar schätze Deleuzes Philosophie Kreativität, dessen Pragmatismus sei sogar eine „,elebration of creativity“ (Thrift, 2000b:276), jedoch fände sich zunehmend Kritik an dessen Auffassung. Nach Thrift macht sich diese Kritik an Deleuzes Philosophie an einer Entleerung der Semantik des Sozialen, einer fehlenden historischen Kontextualisierung und einer Ambivalenz in Hinblick auf binäre Oppositionen fest (Thrift, 2000b:276). Stattdessen verweist Thrift (2000b:272) auf Joas ebenfalls pragmatische Handlungstheorie, die neue Variationen des subjektiven Handelns aus situativen Spannungen heraus begreift, die das Auftreten von Problemen erzeuge (Joas, 1996). Dalton weist darauf hin, dass Joas Kreativität als kontinuierliche Abfolge von Anpassungen an habituelle Schemata des Handelns auffasst, die wiederum lediglich auf die Handlungskontexte reagieren (Dalton, 2004:607). Diese Vorstellung individueller Kreativität wäre damit extrem durch Kontextfaktoren gelenkt wie Institutionen, Normen oder soziale Gruppen. Thrift substituiert an dieser Stelle damit ein poststrukturalistischkollektivistisches Kreativitätsverständnis mit einer enggeführten subjektiven Kreativitätsauffassung und bleibt letztlich in Bezug auf die theoretische Frage, ob Kreativität subjektives oder kollektives Phänomen sei, unentschieden.

Wylie skizziert als zweiten theoretischen Ort der Kreativität in der NRT eine fortwährende kreative Evolution, die sich nicht in binäre Oppositionen von Subjekt/Objekt, lebendig/unlebendig oder Grund/Wirkung auflösen liesse. In dieser poststrukturalistischen Weltbeschreibung bleibt kreatives Handeln immer ein Effekt und wird nie zu einem Grund. Kreativität ist demnach keinem Subjekt zurechenbar, sondern sie stellt eine Assemblage von Flüssen, Netzwerken und Aktanten dar, die konventionelle Grenzen des Menschlichen und des Nicht-Menschlichen desavouiere (Wylie, 2010:105). Eng verwoben mit dieser zweiten Kreativitätsauffassung in der NRT ist das Ereignis als ein Einbruch von Wandel und Neuem in Routinen als ein mögliches Resultat der Konstellation von Flüssen, Netzwerken und Aktanten (Anderson und Harrison, 2010).

Kreativität ist drittens in der NRT mit der Idee des Affekts und der Zirkulation von Affekten verbunden. Kreativität wird in diesem Kontext in körperlichen Bewegungen, Sinneseindrücken und Atmosphären verortet (Wylie, 2010:105). Diese Auffassung von Kreativität ist jedoch abermals nicht frei von Subjektivität, die wiederum über die Bedeutung von Erinnerungen für die Entstehung von Affekten die Kreativität tangiert. Affekte und in deren Folge Kreativität entstehen auf der Basis gelebter Erfahrung als Erinnerung, wie Jones betont. ,[C]reativity emerges not simply from the moment per se, but from the legacies of the past carried into the present, not least through memory which underpins imagination, creativity and (productive) affective exchange" (Jones, 2011:875). Das Subjekt kehrt folglich über die subjektive gelebte Erfahrung, die wiederum eng mit der Kognition verbunden ist (Butcher, 2012:91), erneut in die Kreativitätskonzeption der NRT zurück, da Erfahrungen und Erinnerungen Affekte in ihrer Entstehung beeinflussen können.

Als Fazit zeigt sich, dass es der NRT theoretisch nicht eindeutig gelingt, Kreativität als eine kollektive Semantik zu etablieren, aus der jegliche Subjektivität getilgt ist. Vielmehr verdeutlicht sich in den Arbeiten der NRT, dass die unterschwellige Präsenz des Subjektkonzepts (Barnett, 2012) dafür Sorge trägt, dass zwei der drei theoretischen Anknüpfungspunkte von Kreativität, die Wylie (2010) skizziert, keine reine Kollektivierung des Konzepts leisten können. Die Frage, wie Neues entsteht, wird damit unterschiedlich von verschiedenen Autor_innen in der NRT beantwortet. Es kann sowohl aus der situativen Reaktion subjektiven Handelns resultieren (Thrift, 2000b), als Ereignis und Assemblage von Flüssen, Netzwerken und Aktanten entstehen (Anderson and Harrison, 2010) sowie aus Affekten und Konstellationen von Affekten, die wiederum nicht unbeeinflusst von Erfahrungen und damit dem Kognitiven (Butcher, 2012:91) sind, hervorgehen (Jones, 2011).

\section{Synthese: PSF und NRT}

Die Ausgangsfrage des Beitrags, ob Kreativität ein subjektiv gebundenes oder ein kollektives Phänomen darstellt, wird in den zwei diskutierten Theorien unterschiedlich beantwortet. In der PSF ist Kreativität als kognitive Aktivitäten von Subjekten konzipierbar (Verene, 1978), die Sinnliches und Sinn dahingehend verknüpfen, dass diese Kognitionsleistungen über die symbolischen Formen in Kultur überführt werden. Die PSF optiert damit für die individuelle Perspektive und steht in Einklang mit den zeitgenössischen Kreativitätsmodellen von Wallas (1926) und Whitehead (1979). Der NRT gelingt es dagegen nicht, in ähnlich überzeugender Weise eine Kreativitätsauffassung zu entwickeln. Kreativität ist zunächst als Affekt, als Assemblage von Netzwerken und Aktanten, als Ereignis und als kreatives Handeln unter Kontextbedingungen konzipiert und folglich ein kollektives Phänomen. Die Entstehung von Neuem ist damit allerdings nicht eindeutig definiert, sondern es schwingt in verschiedenen Konzepten lediglich implizit mit. Darüber hinaus 
führen Affekte und kreatives Handeln das Subjekt und damit das Mikro-Makro-Verhältnis unterschwellig mit. Eine theoretisch konsistente Konzipierung der kreativen Praxis (Thrift, 2000a:213) legt die NRT folglich nicht vor.

Auf der Basis des bisher Gesagten bieten sich zwei Möglichkeiten an, über die PSF die misslingende radikale Kollektivierung der Kreativitätssemantik in der NRT zu mildern und damit der Entstehung von Neuen einen theoretischen Ort zuzuweisen. Die erste Möglichkeit bietet die Soziologie Bourdieus, die als eine Soziologisierung von Teilen der cassirerschen Philosophie interpretierbar ist. Die Figur der Inkorporierung gesellschaftlicher Verhältnisse als Habitus des Akteurs wird von Thrift als bedeutender Impuls für die NRT betrachtet (Thrift, 1996:2). Bourdieu führt mit dem Habitus die Idee aus, dass sich im zeitlichen Verlauf einer Biographie die gesellschaftlichen Bedingungen in die Körper einschreiben, der von Akteuren frei gestaltbare Lebensverlauf mithin zu einer ,Illusion“ gerät (Bourdieu, 1998:75-83). Diese Inkorporierung sozialer Beschränkungen ist wiederum ein wesentlicher Aspekt der nonrepresentationalen Praxis (Cresswell, 2002:380). Der Habitus als Einschreibung gesellschaftlicher Verhältnisse erhebt den Körper zum Symbol des Gesellschaftlichen und fungiert damit als Äquivalent zur symbolischen Prägnanz als Zusammenhang von Sinnlichem und Sinn in der PSF (Bickel, 2003:114).

Individuelle Kreativität setzt an der Stelle der Verknüpfungsleistung dieser Symbolisierung an. Die Interpretation dieser in der Symbolisierung verborgenen Beziehung von Individuellem bzw. Sinnlichem und Kollektiven bzw. Sinn ist in der PSF ein kreativer Akt, für Bourdieu hingegen das Resultat der Wirkung gesellschaftlicher Macht auf die Subjekte, die sich gerade in den symbolischen Formen zeige, da diese nie frei von Interessen seien (Bourdieu, 1998:121). Die NRT könnte an dieser Stelle auf die cassirerschen Grundlagen der bourdieuschen Einschreibungsmetapher zurückgreifen und den Zusammenhang von Sinnlichem und Sinn weniger im Kontext kollektiver gesellschaftlicher Macht- und Herrschaftsverhältnisse als Inkorporierung auffassen, sondern in einem stärker cassirerschen Sinne als ein Element der Differenzierung zwischen dem Individuellen und dem Allgemeinen. Die Kreativitätssemantik in der NRT wäre damit nicht länger durch den Machtbegriff bei Bourdieu blockiert. Vielmehr firmiert Kreativität als Vermittlerin zwischen der nonrepresentationalen Praxis, die von menschlichen und nichtmenschlichen Körpern und damit Symbolen ausgeführt wird und einen Sinnzusammenhang symbolischer Formen. Inkorporierung ist damit nicht bedeutungslos, sondern erklärt die Auffassung von Körpern als Symbole. Kreativität sorgt nun für die Verknüpfung des sinnlich-materiellen Aspekts der Körper mit dem ideellen Sinn dessen, was sie symbolisieren. Damit wird die nonrepresentationale Praxis zu einer ,creative praxis“ (Thrift, 2000a:213). Kreativität wäre mit diesem Vorschlag individuell mit denjenigen Akteuren und Aktanten verbunden, die als Symbole an der nonrepresentationalen
Praxis teilhaben, aber nicht notwendigerweise auf ein Subjekt angewiesen.

Eine zweite Möglichkeit der theoretischen Einbettung einer individuellen Kreativitätsauffassung in der NRT liefert die Affektsemantik. Der Affektbegriff ist einer der umstrittensten (z.B. Leys, 2011; Korf, 2012) und komplexesten Vokabeln der NRT. Affekt wie Gefühl werden hier u.a. als inkorporierte Phänomene verstanden. „,Affects] act on bodies, are produced through bodies and transmitted by bodies“ (Lorimer, 2008:552). Das kreative Potenzial von Affekten wird in der NRT erkannt, so Jones (2011). Sie übersähe aber die Bedeutung der gelebten Erfahrung der Körper als Erinnerung. Damit werden Affekte zugleich mit Kognition verbunden, da die Existenz einer im Kontext des Körpers festzumachenden Grenze zwischen dem Kognitiven und der Erfahrung von den Kognitionswissenschaften bezweifelt wird (Butcher, 2012:91). Erinnerung und Erfahrung bilden die Brücke zwischen Affekt in der NRT und Affekt und Gefühl in der PSF.

Der Affekt als Gefühl ist in der Philosophie Cassirers dagegen ein „passives Erleben“ (Schwemmer, 1997:134). Damit ist zugleich aber nicht gesagt, dass Gefühl und Affekt keine geistigen Ereignisse oder differenzierende Gestaltungen seien. Affekte und Gefühle sind Teil des kollektiven geistigen Lebens und nicht dem abgegrenzten Bereich des Bewusstseins vorbehalten (Schwemmer, 1997:134). Sie sind damit genauso mit Erfahrung und Erinnerung verbunden, wie die Affektsemantik der NRT dies implizit ebenfalls ist. Der aus der PSF entwickelte individuelle Kreativitätsbegriff als eine Verknüpfung von Sinnlichem mit Sinn, die über symbolische Formen objektiviert wird, ist folglich auch in Gefühlen und Affekten involviert. Individuelle Kreativität ist theoretisch bereits an der Entstehung von Affekten und Gefühlen beteiligt, wenn diese auf Erfahrung und Erinnerung beruhen und als Folge der Äußerungen dieser Affekte und Gefühle kann wiederum Kreativität in einem kollektiven Sinne entstehen (Hasse, 2003:27-28).

Der Beitrag kann abschließend nicht entscheiden, ob es sich bei der Kreativitätssemantik um ein subjektiv gebundenes oder kollektives Phänomen handelt. Vielmehr entwickelt er mithilfe der PSF zunächst eine Vorstellung individueller Kreativität, die an eingangs des Beitrags diskutierte Konzepte der Psychologie anschließt. Diese Kreativitätsauffassung ist anschließend in der Lage, zwei Vorschläge für die NRT $\mathrm{zu}$ unterbreiten, um einen theoretisch konsistenten Ort für die Entstehung von Neuem in dieser humangeographischen Theorie zu konzipieren. Kreativität lässt sich zum einen über Bourdieus Figur der Inkorporierung mit der nonrepräsentionalen Praxis verbinden. Zum anderen bietet eine Lesart, die Affekt mit Erfahrung kombiniert, einen theoretischen Ort für Kreativität in der NRT. Welcher dieser zwei Vorschläge zur Milderung des Mikro-Makro-Problems in der NRT der gangbarere ist, müsste empirische Forschung in Zukunft zeigen. 
Acknowledgements. Ich danke Ilse Helbrecht, Benedikt Korf, Catherine Robin, Wolf-Dietrich Sahr, Barbara Zahnen und drei anonyme Gutachter_innen für wertvolle Hinweise und Anregungen, die diesen Aufsatz in der vorliegenden Form erst ermöglichten.

Edited by: B. Korf

Reviewed by: three anonymous referees

\section{Literatur}

Amabile, T. M.: Creativity in context, Westview Press, Boulder, Oxford, 1996.

Anderson, B. and Harrison, P.: The promise of non-representational theories, in: Taking-place: non-representational theories and geography, edited by: Anderson, B. and Harrison, P., Farnham, Burlington, 1-34, 2010.

Barnett, C.: Geography and ethics: placing life in the space of reasons, Prog. Hum. Geogr., 36, 379-388, 2012.

Bickel, C.: Die Cassierer-Rezeption bei Bourdieu, in: Kunst, Macht und Institution. Studien zur Philosophischen Anthropologie, soziologischen Theorie und Kultursoziologie der Moderne, Herausgeber: Fischer, J. und Joas, H., Frankfurt am Main, 111-118, 2003.

Bourdieu, P.: Die feinen Unterschiede. Kritik der gesellschaftlichen Urteilskraft, Suhrkamp, Frankfurt am Main, 1987a.

Bourdieu, P.: Sozialer Sinn. Kritik der theoretischen Vernunft, Suhrkamp, Frankfurt am Main, 1987b.

Bourdieu, P.: First lecture. Social space and symbolic space: introduction to a Japanese reading of distinction, Poetics Today, 12, 627-638, 1991.

Bourdieu, P.: Praktische Vernunft. Zur Theorie des Handelns, Suhrkamp, Frankfurt am Main, 1998.

Bourdieu, P.: Meditationen. Zur Kritik der scholastischen Vernunft, Suhrkamp, Frankfurt am Main, 2001.

Butcher, S.: Embodied cognitive geographies, Prog. Hum. Geogr., 36, 90-110, 2012.

Cassirer, E.: Goethe und die geschichtliche Welt. Drei Aufsätze, Verlag Bruno Cassirer, Berlin, 1932.

Cassirer, E.: The concept of group and the theory of perception, Philos. Phenomen. Res., 5, 1-36, 1944.

Cassirer, E.: Philosophie der symbolischen Formen. Erster Teil. Die Sprache, Wissenschaftliche Buchgesellschaft, Darmstadt, 1994a.

Cassirer, E.: Die „Tragödie der Kultur“, in: Zur Logik der Kulturwissenschaften. Fünf Studien, Cassirer, E., Darmstadt, 103-127, 1994b.

Cassirer, E.: Das Symbolproblem und seine Stellung im System der Philosophie, in: Ernst Cassirer. Symbol, Technik, Sprache. Aufsätze aus den Jahren 1927-1933, Herausgeber: Orth, E. W. und Krois, J. M., Hamburg, 1-21, 1995.

Cassirer, E.: Der Begriff der symbolischen Form im Aufbau der Geisteswissenschaften, in: Ernst Cassirer. Schriften zur Philosophie der symbolischen Formen, Herausgeber: Lauschke, M., Hamburg, 63-92, 2009.

Cassirer, E.: Philosophie der symbolischen Formen. Dritter Teil. Phänomenologie der Erkenntnis, Felix Meiner Verlag, Hamburg, 2010.

Chen, K. K.: Organizing creativity: enabling creative output, process, and organizing practices, Sociology Compass, 6, 624-643, 2012.
Cresswell, T.: Bourdieu's geographies: in memorium, Environ. Plann. D, 20, 379-382, 2002.

Csikszentmihalyi, M.: Motivation and creativity: toward a synthesis of structural and energistic approaches to cognition, New Ideas Psychol., 6, 159-176, 1988.

Csikszentmihalyi, M.: Creativity. Flow and the psychology of discovery and invention, HarperPerennial, New York, 1997.

Dewsbury, J.-D., Harrison, P., Rose, M., and Wylie, J.: Enacting geographies, Geoforum, 33, 437-440, 2002.

Dalton, B.: Creativity, habit, and the social products of creative action: revising Joas, incorporating Bourdieu, Sociol. Theor., 22, 603-622, 2004.

Grabher, G.: Ecologies of creativity: the Village, the Group, and the heterarchic organisation of the British advertising industry, Environ. Plann. A, 33, 351-374, 2001.

Guilford, J. P.: Creativity, Am. Psychol., 5, 444-454, 1950.

Guilford, J. P.: The nature of human intelligence, McGraw-Hill Book Company, New York, 1967.

Hasse, J.: Die Frage nach den Menschenbildern - eine anthropologische Perspektive, in: Menschenbilder in der Humangeographie, Wahrnehmungsgeographische Studien, 21, Herausgeber: Hasse, J. und Helbrecht, I., Oldenburg, 11-31, 2003.

Heintz, B.: Emergenz und Reduktion. Neue Perspektiven auf das Mikro-Makro-Problem, Kölner Z. Soziol. Soz., 56, 1-31, 2004.

Heusden, B. v.: Cassirers Ariadnefaden - Anthropologie und Semiotik, in: Kultur und Symbol. Ein Handbuch zur Philosophie Ernst Cassirers, Herausgeber: Sandkühler, H. J. und Pätzold, D., Stuttgart, Weimar, 111-147, 2003.

Hoel, A. S.: Thinking "difference" differently: Cassirer versus Derrida on symbolic mediation, Synthese, 179, 75-91, 2011.

Joas, H.: The creativity of action, University of Chicago Press, Chicago, 1996.

Jones, O.: Geography, memory and non-representational geographies, Geography Compass, 5, 875-885, 2011.

Kaufman, J. C. and Beghetto, R. A.: Beyond big and little: the four C model of creativity, Rev. Gen. Psychol., 13, 1-12, 2009.

Korf, B.: Neuro-Kulturgeographie, Geogr. Z., 100, 146-163, 2012.

Krois, J. M.: Ernst Cassirers Semiotik der symbolischen Formen, Z. Semiotik, 6, 433-444, 1984.

Krois, J. M.: Problematik, Eigenart und Aktualität der Cassirerschen Philosophie der symbolischen Formen, in: Über Ernst Cassirers Philosophie der symbolischen Formen, Herausgeber: Braun, H.-J., Holzhey, H., und Orth, E. W., Frankfurt am Main, 15-44, 1988.

Leys, R.: The turn to affect: a critique, Crit. Inquiry, 37, 434-472, 2011.

Lorimer, H.: Cultural geography: non-representational conditions and concerns, Prog. Hum. Geogr., 32, 551-559, 2008.

Magerski, C.: Die Wirkungsmacht des Symbolischen. Von Cassirers Philosophie der symbolischen Formen zu Bourdieus Soziologie der symbolischen Formen, Z. Soziol., 34, 112-127, 2005.

Mersch, D.: Imagination, Figuralität und Kreativität. Zur Frage der Bedingungen kultureller Produktivität, in: Kreativität (Kolloquienbeiträge). XX. Deutscher Kongress für Philosophie, Herausgeber: Abel, G., Hamburg, 344-359, 2006.

Meusburger, P.: Milieus of creativity: the role of places, environments, and spatial contexts, in: Milieus of creativity. An interdisciplinary approach to spatiality of creativity, Knowledge and 
Space, 2, Herausgeber: Meusburger, P., Funke, J., and Wunder, E., Heidelberg, 97-153, 2009.

Neher, A.: How perspective could be a symbolic form, J. Aesthet. Art Critic., 63, 359-373, 2005.

Osborne, T.: Against "creativity": a philistine rant, Econ. Soc., 32, 507-525, 2003.

Paetzold, H.: Ernst Cassirer zur Einführung, Junius, Hamburg, 2008.

Rhodes, M.: An analysis of creativity, Phi Delta Kappan, 42, 305$310,1961$.

Schwemmer, O.: Ernst Cassirer. Ein Philosoph der europäischen Moderne, Akademie Verlag, Berlin, 1997.

Taggar, S.: Individual creativity and group ability to utilize individual creative resources: a multilevel model, Acad. Manage. J., 45, 315-330, 2002.

Thrift, N.: Spatial Formations, Sage Publications, London, 1996.

Thrift, N.: The still point. Resistance, expressive embodiment and dance, in: Geographies of resistance, edited by: Keith, M. and Pile, S., London, New York, 124-151, 1997.

Thrift, N.: Steps to an ecology of place, in: Human geography today, edited by: Allen, J., Massey, D., and Sarre, P., Cambridge, 295$322,1999$.
Thrift, N.: Afterwords, Environ. Plann. D, 18, 213-255, 2000a.

Thrift, N.: Entanglements of power. Shadows? in: Entanglements of power. Geographies of domination/resistance, edited by: Sharp, J. P., Routledge, P., Philo, C., and Paddison, R., London, New York, 269-278, 2000b.

Thrift, N.: Knowing capitalism, Sage Publications, London, 2005.

Thrift, N.: Donna Haraway's dreams, Theor. Cult. Soc., 23, 189195, 2006.

Thrift, N.: Non-representational theory. Space, politics, affect, Routledge, London, 2008.

Törnqvist, G.: Creativity in time and space, Geogr. Ann. B., 86, 227-243, 2004.

Verene, D. P.: Kant, Hegel, and Cassirer: the origins of the philosophy of symbolic forms, J. Hist. Ideas, 30, 33-46, 1969.

Verene, D. P.: Cassirer' s concept of symbolic form and human creativity, Idealistic Stud., 8, 14-32, 1978.

Wallas, G.: The art of thought, Harcourt, Brace, New York, 1926.

Whitehead, A.: Process and reality. An essay in cosmology, The Free Press, New York, 1979.

Wylie, J.: Non-representational subjects? in: Taking-place: nonrepresentational theories and geography, edited by: Anderson, B. and Harrison, P., Farnham, Burlington, 99-114, 2010. 